\title{
QCD phases at high density and instantons
}

\author{
E.Shuryak ${ }^{\mathrm{a}}$
}

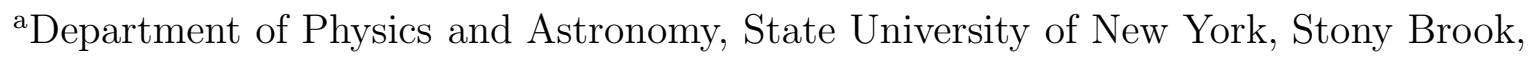
NY 11794-3800

The talk is an introduction into diquark condensation phenomena which occur in QCD at high energy density. They are driven by instantons and instanton-antiinstanton pairs (or "molecules"), which generate attraction in some qq channels. A number of phases is possible, with or without restoration of chiral symmetry: the work is not finished and we do not yet know which take place in real QCD. We also emphasize that specific diquark correlations play a significant role in baryon structure, in particular making that of a nucleon very different of a $\Delta$ (or other member of a decuplet). This "small $N_{c}$ " scenario based on comparison to QCD with two colors is contrasted with the "large $N_{c}$ " one.

\section{INTRODUCTION}

At this workshop one probably does not need to explain why we study QCD at large density: it is, after all, a traditional domain of nuclear physics. However, we now discuss it in quite different perspective, as a part of recent development related with the QCD transitions into what is generally known as the quark-gluon plasma (QGP).

We know much more about high temperature QCD and the corresponding transitions, mostly because of systematic use of our main tool, the lattice QCD. Numerical simulations have shown that the main non-perturbative features of the QCD vacuum - confinement and chiral symmetry breaking - do indeed disappear in a common high temperature phase transition, at $T=T_{c} \sim 150 \mathrm{MeV}$. The debates about its mechanism is a focal point of non-perturbative QCD: all vacuum models (using instantons, abelian projected monopoles or $Z_{N}$ vortices or any other beasts) claim to explain it. The same is claimed by many field theory models (e.g. the NJL model) or even the resummed perturbative one-gluon exchange! However, in order to see which claims are in fact true many significant details of the picture should still be clarified. Let me on the onset of the talk mention few of them, first at qualitative and then quantitative level.

Among qualitative questions people mostly discussed whether their favorite objects mentioned above show any change at $T \approx T_{c}$. I think the main issues to address now are dependence of the finite $\mathrm{T}$ transitions on the number of quark flavors $N_{f}$ and their masses. The boundary of the unknown here is not so far out: we do not know what happens for chiral phase transition for as small number of of flavors as $N_{f} \sim 5$, where the usual instantonbased mechanism of chiral symmetry breaking seems to fail and instanton-antiinstanton "molecules" were predicted to dominate even the vacuum state (see [6]). What happens between this number and $N_{f}=11 N_{c} / 2$ (where asymptotic freedom ends) is still a matter 
of speculations. Amusingly, we now know the phase diagram of super-symmetric QCD much better, due to famous works of Seiberg and collaborators. If anything, we have learned from them that fermions are important, and can lead to many different phases. Another set of qualitative questions deal with hadrons/correlation functions at $T>T_{c}$. In particular, there are intriguing deviations of pion-sigma "screening masses" from vector ones: can those be reproduced by all those models?

One can certainly ask lots of questions at the quantitative level, but still let me point out only one important point which is rarely discussed. Why is the phase transition in "quenched QCD" $\left(N_{f}=0\right) T_{\text {quenched }} \sim 260 \mathrm{MeV}$ is so much higher than $T_{N_{f}=2} \sim$ $150 \mathrm{MeV}$ ? How exactly the hadronic spectrum in these case/or fermionic determinant for particular configurations leads to this smaller critical temperature?

Much less work has been done in the field of finite density QCD. Since the main perturbative phenomena (such as screening and plasma oscillations) in dense quark matter has been worked out (see e.g. [1]), the field was essentially dormant for 2 decades. The lattice approach to it was much discussed at this meeting, and it is probably still true that so far this approach does not work for finite chemical potential. The issue is usually discussed in connection with complex fermionic determinant, to which standard MonteCarlo algorithms do not apply, and people try to avoid it. However, other methods also start from the vacuum ensembles, trying to deduce properties of high density matter from them. Very small overlap between the two leads to enormous numerical problems: what is needed is some way to simulate the high density matter by itself.

Being short of such method, we use the well-tested analytic methods such as mean field (MF) and Random Phase Approximation (RPA). They have done a good job for the usual super-conductivity, or pairing correlations in nuclei. As the Coulomb interaction between quarks of different colors is attractive, it was realized early on that Cooper pairs can be formed and condense, making the quark plasma a super-conductor. The magnitude of the corresponding gap $\Delta$ and the critical temperature $T_{c}$ were estimated to be in the 1 $\mathrm{MeV}$ range [2].

Recent interest to this problem was revived by two groups [3, [4] which independently came from two different angles to a very similar conclusions. They have shown that instantons may generate much stronger "color super-conductor", with a gap in the $100 \mathrm{MeV}$ range. Specifically, these works dealt with the simplest 2-flavor theory, in which only one basic type of diquarks, $\mathrm{I}=0$ or ( $\mathrm{ud}-\mathrm{du}$ ) can be formed, in anti-triplet color representation. After the original papers further progress include interesting new phases including strange quarks [5]. The issue here is whether a gap of similar magnitude can be generated by instantons, or concentrate instead on smaller gap generated by the one gluon exchange (OGE).

New rapidly growing field or research is related to the vicinity of the tricritical point [28,29,26]. Those resemble the well known liquid-gas nuclear transition much studied in low energy heavy ion collisions. Hopefully, it will create equally fruitful experimental works!

At the end of the introduction let me say that we now see only the tip of the iceberg. Diquark condensation is fighting against the chiral condensate of different nature, and probably also against the mesonic condensates discussed previously [13]. On top of it there appear instanton clusters (molecules), another kind of clustering which is also fermion- 
driven but do not need any condensates at all. These three phenomena make a triangle of love-hate relationships not less complicated than in a classic novel. It will take much more work to sort it out.

\section{WHY INSTANTONS?}

Instantons are classical solutions of Yang-Mills equations, describing the tunneling paths from one minimum of the potential to the other. As discussed e.g. in recent review [6], instantons are the strongest non-perturbative phenomena in QCD. In particularly, when lattice configurations are made somewhat more smooth (removing quantum noise corresponding to perturbative high-frequency fields), it is instantons which they mainly see. Therefore, their density is related to the value of the gluon condensate. Furthermore, it is instantons which seem to generate the famous $1 \mathrm{GeV}$ "chiral Lagrangian" scale円 , separating perturbative and non-perturbative domains (see recent discussion of this issue in [7]). Instanton-based models quantitatively explain chiral symmetry breaking and pion properties, and even spectroscopy of lowest mesons and baryons (including $N, \Delta$ ). Although they by no means include all non-perturbative phenomena (e.g. they produce too weak confining potential), they seem to be sufficient for description of all phenomena relevant for traditional nuclear physics.

Instantons are present not only in QCD, and arguments for their dynamical role are not only empirical. As a good theoretical example let me mention $\mathrm{N}=2$ SUSY QCD, for which Witten and Seiberg have found exact effective Lagrangian [8]. As the photon sector of the theory has only one-loop perturbation theory, the Witten-Seiberg solution in weak coupling is nothing but a series of the instanton-generated diagrams. Instantons blow up the coupling constant at a scale $2^{3 / 2} \Lambda_{\text {pert }}$, with an amusing numerical coincidence with real QCD [7].

The main reason instantons are important in QCD is because of their relation to anomaly and light fermions. A general topological argument demands that tunneling produce certain rearrangement in fermionic sector: this leads to effective interactions we use. It is very important that these general arguments are also valid in high density matter. In particular, the famous explanation of the anomaly as fermion level motion (known also as an "infinite hotel" story) can be directly applied to cold quark matter. An obvious conclusion is that "extra" fermion pairs produced by tunneling must show up in the same multiplicity as in vacuum, but now at the Fermi surfacef? This consideration alone suggests interesting instanton-induced effects at high density.

\section{QCD WITH ONLY TWO COLORS}

We start with this theory for several reasons. The main one is pedagogical: it will teach us some important lessons about diquark spectroscopy. The secondary one is that it is a very special case, in which simple theoretical and even lattice studies of the finite

\footnotetext{
1 Note that this scale was for a long time puzzling to people doing perturbative QCD: at 1 GeV nothing bad happens with the perturbative running coupling constant.

${ }^{2}$ In random matrix models people have used a simple-minded approach: adding $\mu \gamma_{4}$ to the Dirac operator represented as a random matrix with some density of zero modes (leading to quark condensate). If $\mu$ is sufficiently large, eigenvalues move away from zero and chiral symmetry gets restored.
} 
$\mu$ case can be done. This theory is in many ways the opposite to the large $N_{c}$ limit, and thus is very instructive to keep it in mind. For large $N_{c}$ baryons are supposed to be $N_{c}$ times heavier than mesons. In the $N_{c}=2$ QCD baryons (or diquarks) are in fact degenerate with mesons, due to Pauli-Gursey symmetry[(PGSY) [9], relating quarks and anti-quarks. In particular, the lowest baryons (or diquark) should be bound as strongly as the lowest meson, the massless pions. The general pattern of symmetry breaking is $S U\left(2 N_{f}\right) \rightarrow S p\left(2 N_{f}\right)$ and the number of Goldstone modes is $N_{\text {goldstones }}=2 N_{f}^{2}-N_{f}-1$. Let us mention two cases specifically. For $N_{f}=1$ there remains no goldstones because there is no symmetry to be spontaneously broken. (Recall that due to U(1) anomaly, even the corresponding meson, $\eta^{\prime}$, is massive.) For the most interesting case $N_{f}=2$ the coset (ratio) of full group over remaining one is

$$
K=S U(4) / S p(4)=S O(6) / S O(5)=S^{5}
$$

which means the 5 -dim sphere with 5 massless modes: three of those are pions, plus scalar diquark $\mathrm{S}$ and its anti-particle $\bar{S}$. At finite but small density one finds that rotations on the 5-dim sphere cost no energy, and by doing so in the direction of the scalar diquark condensate one naturally obtains states with non-zero diquark density. So the critical point is $\mu=0$ (for a non-zero quark mass it is half the pion mass), and the usual chiral condensate $\langle\bar{q} q>$ is rotated into a diquark one. The qualitative picture can be understood using the corresponding linear sigma model. The potential

$$
V=\lambda\left(\vec{\pi}^{2}+\sigma^{2}+S^{2}+\bar{S}^{2}-v^{2}\right)^{2}-A \sigma-\mu^{2}\left(S^{2}+\bar{S}^{2}\right)
$$

includes the diquark chemical potential $\mu$ and the chirally asymmetric mass term $A$. At $\mu=0$ the Goldstone masses are $m_{g}^{2}=A / v$, and $m_{\sigma}^{2}=8 \lambda v^{2}$. For non-zero $\mu$ we can determine the $\langle\bar{q} q\rangle$ and $\langle q q\rangle$ condensates $\langle\sigma\rangle$ and $\langle S\rangle$ using the mean field approximation. We find

$$
4 \lambda\langle S\rangle\left(\sigma^{2}+\langle S\rangle^{2}-v^{2}\right)=2 \mu^{2}\langle S\rangle .
$$

Below the critical chemical potential $\mu_{c} \simeq m_{g} / \sqrt{2},\langle\sigma\rangle$ is constant and $\langle S\rangle=0$. Above $\mu_{c},\langle S\rangle$ increases as

$$
\langle S\rangle^{2}=\frac{\mu^{2}}{2 \lambda}+v^{2}-\frac{A^{2}}{4 \mu^{2}}
$$

and $\langle\sigma\rangle=m_{g}^{2} v /\left(2 \mu^{2}\right)$. The energy density is $\epsilon=-\mu^{2} v^{2}-3 A^{2} /(4 \mu)^{2}$, compared to $\epsilon=-m_{g}^{2} v^{2}+m_{g}^{4} /(16 \lambda)$ for the normal vacuum.

Unlike real QCD, $N_{c}=2$ gauge theory is straightforward to simulate on the lattice, since the fermion determinant remains real for $\mu \neq 0$. With the exception of some early work using small lattices and the strong coupling expansion [10], few studies have taken advantage of this. Numerical studies of the instanton model for $N_{c}=2$ at finite density have been done in 11. All of them display how at large density the chiral $\langle\bar{q} q\rangle$ condensate is replaced by the diquark one $\langle q q\rangle$, in agreement with the sigma model described above.

3 Unlike in SUSY, in this case there are different number of baryons and mesons in multiplets, and both are of course bosons. 


\section{DIQUARKS IN REAL QCD (WITH 3 COLORS)}

Before we return to the real world, we review properties of the instanton-induced interaction due to 't Hooft, rewritten in $\bar{q} q$ and $q q$ channels. We consider only the simplest case of two flavors (up and down). As a shorthand notations, effective Lagrangian can be written as follows

$$
\mathcal{L}=G \frac{1}{8 N_{c}^{2}}\left[\left(\bar{\psi} \tau^{-} \psi\right)^{2}+\left(\bar{\psi} \tau^{-} \gamma_{5} \psi\right)^{2}\right],
$$

where we have added the interaction in the direct and exchange channels and dropped color octet terms. $N_{c}$ is the number of colors and $\tau^{-}=(\vec{\tau}, i)$ is an isospin matrix. In this way we have combined isospin 1 channel (including the pion) with the isospin 0 one (denoted as $\eta^{\prime}$ ). The i squared leads to a sign difference for them, showing that the same interaction tends to make pion light and $\eta^{\prime}$ heavy. The coupling $\mathrm{G}$ is related to the instanton density and we would not discuss it here, except to comment that the huge magnitude of the $\pi-\eta^{\prime}$ splitting hints once again about large scale of the instanton-induced effects.

In this discussion we are actually interested in another manifestation of this Lagrangian, in the diquark channel. Its phenomenological implications in this case were first discussed in connection with spin-dependent forces in baryons [16, challenging the conventional wisdom that spin splittings in baryons are due to the one-gluon exchanget. The same Lagrangian (5) can be Fierz-rearranged into a $(q q)$ interaction:

$$
\begin{aligned}
& \mathcal{L}= G\left\{-\frac{1}{16 N_{c}\left(N_{c}-1\right)}\left[\left(\psi^{T} C \tau_{2} \lambda_{A}^{n} \psi\right)\left(\bar{\psi} \tau_{2} \lambda_{A}^{n} C \bar{\psi}^{T}\right)\right.\right. \\
&\left.+\left(\psi^{T} C \tau_{2} \lambda_{A}^{n} \gamma_{5} \psi\right)\left(\bar{\psi} \tau_{2} \lambda_{A}^{n} \gamma_{5} C \bar{\psi}^{T}\right)\right] \\
&\left.+\frac{1}{32 N_{c}\left(N_{c}+1\right)}\left(\psi^{T} C \tau_{2} \lambda_{S}^{n} \sigma_{\mu \nu} \psi\right)\left(\bar{\psi} \tau_{2} \lambda_{S}^{n} \sigma_{\mu \nu} C \bar{\psi}^{T}\right)\right\}
\end{aligned}
$$

Here, $C$ is the charge conjugation matrix, $\tau_{2}$ is the anti-symmetric Pauli matrix, $\lambda_{A, S}$ are the anti-symmetric (color $\overline{3}$ ) and symmetric (color 6) color generators. This Lagrangian (6) provides a strong attractive interaction between an up and a down quark with antiparallel spins $\left(J^{P}=0^{+}\right)$in the color anti-triplet channel, and a repulsive interaction in the $0^{-}$channel. $0^{+}$quark pairs couple to the diquark current $S_{d q}^{a}=\epsilon_{a b c} u_{b}^{T} C \gamma_{5} d_{c}$.

Quantitative studies of instanton effects in baryon spectroscopy, both for light and heavy-light systems, were done in [17]. It was first found that instanton interaction alone not only makes the nucleons and deltas to be bound states of constituent quarks, but even their splitting also came out right (being even somewhat larger than the observed one). Further studies have found that the nucleon has a very large overlap with the current $\epsilon_{a b c}\left(u_{a}^{T} C \gamma_{5} d^{b}\right) u^{c}=S_{d q}^{a} u^{a}$, containing scalar diquark. Both observation were clarified by further analysis, in which either the third quark is taken to be infinitely heavy (and thus contributing only the color phase matrix), or fixing the gauge and ignoring the third

\footnotetext{
${ }^{4}$ Of course, even its strong proponents should explain where magnetic moment of a quark comes from. Obviously it must be the same mechanism which generates the constituent quark mass, and therefore we are back to instantons.
} 
quark completely. Either way one finds a deeply bound scalar diquark (Sdq) $2 m_{q}-m_{S d q} \simeq$ $200-300 \mathrm{MeV}$, whereas in all other channels (vectors and axial-vectors, color 6 diquarks, etc.) no such binding was found. Furthermore, one can derive the diquark binding analytically, in the usual RPA approximation?. The first lattice attempt 15 to study "diquark spectroscopy" have indeed found that a scalar diquark is more bound than vector one, but the effect is rather modest ${ }^{\circ}$.

Note a nice continuity at this point: while changing from $N_{c}=2$ to 3 colors the same scalar diquark goes from being nearly massless (bound by about $2 M_{\text {const }} \sim 700 \mathrm{MeV}$ ) to a deeply bound state. It can be traced to the coefficients of the Lagrangian given above: for $N_{c}=2$ the corresponding coupling constants in mesonic and diquark channels are equal, while they become factor 2 different for $N_{c}=3$.

Let us qualitatively compare the "large $N_{c}$ " picture of baryons with our "small $N_{c}$ ". The former suggests a picture of baryons as large heavy objects, made of classical pion fields. In it $N, \Delta$ (and other members of octet and decuplet) are basically the same object, slowly rotating with a slightly different angular momenta. The latter picture is radically different: the octet baryons can be pictured as a strongly bound (and spatially compact) scalar diquark plus the third quark. The decuplet baryons do not have scalar diquarks, and are therefore generically 3-body objects. Which one is closer to the real world? A long list of phenomenological hints suggesting the quark-diquark picture is better can be found in a review [27].

One particularly important observation is strikingly different behavior of the electromagnetic form-factor of the $N \rightarrow \Delta$ transition, as compared to $N \rightarrow N^{*}$ (and also elastic one): The former is decreasing with $Q^{2}$ much quicker than the latter. It is difficult to get such behavior, unless the structure of $N$ and $\Delta$ are very different.

Let me add one more hint to the list $[$. Although in order to be convinced by it one still has some experimental work to do, it is rather general and model-independent. It is related to a phenomenon of inelastic diffraction in high energy hadronic collisions. As noticed by Good and Walker already in 1960, it exists due to different absorption probability of different component in hadronic wave functions. (If all of them be absorbed equally, the only diffraction left would be the elastic one.) Data on $\pi$ and $\mathrm{N}$ inelastic diffraction of nuclear targets have been analyzed in [19], and the corresponding distributions $P(\sigma)$ over fluctuating cross sections $\sigma$ were derived. Amazingly, the one for the nucleon is as wide (if not wider) than that for the pion. Let me speculate that this wide distribution is due to quasi-two-body (quark-diquark) structure of a nucleon. The opposite large- $N_{c}$-inspired picture, a skyrmion, should on the contrary lead to small fluctuations of essentially classical object?. Can one check this interpretation experimentally? Unfortunately there can

\footnotetext{
${ }^{5}$ The opposite conclusion reached in $[18]$ is due to summation of only part of the needed diagrams.

${ }^{6}$ We hope future data at smaller quark masses will provide more accurate extrapolation to physical masses: the present data (plus linear extrapolation in quark mass) gives only about half of the nucleondelta mass splitting. Note also, that the qualitative difference between nucleon and delta correlators at intermediate distances found in the instanton model [17] were confirmed on the lattice by the MIT group 12].

'I am indebted to M.Strikman, who brought this interesting works to my attention.

8 Large observed fluctuations of $\sigma$ also contradict to another a priori possible picture, is that of a large number $\mathrm{N}$ of partons. If those were to interact independently, one should expect relatively small fluctuations of $\sigma, \sim 1 / N^{1 / 2}$.
} 
be no beams of $\Delta$ particles. However, there are those for another decuplet member, the celebrated $\Omega^{-}$. What size are fluctuations of $i t s$ cross section on nuclei? Is it really smaller than that for the nucleon?

\section{INSTANTON EFFECTS AT HIGH DENSITY: VARIOUS PHASES}

The problem we are going to discuss is an ensemble of quarks coupled to an ensemble of instantons. Like in so many other problems of statistical mechanics, we find one more case of a competition between order and disorder, or energy versus entropy. The QCD vacuum has strongly disordered instantons, with nearly random distributions and non-zero chiral condensate. Mean field analysis seem to be quite adequate in this case: it suggests a simple picture that at whatever space-time point the coupling occurs, the fermion "legs" of the Lagrangian given above can be simply absorbed by the vacuum condensates. Curiously enough, the QGP phase at high T is made of ordered (or clustered) instantons, combined into so called instanton-anti-instanton molecules [20,17]. They have been predicted to be directed in time direction, and there are first lattice data [23] showing that at $T \sim T_{c}$ this is indeed the case. There are no condensates available in the usual QGP phase, and so (massless) quarks has to propagate from instanton to anti-instantons.

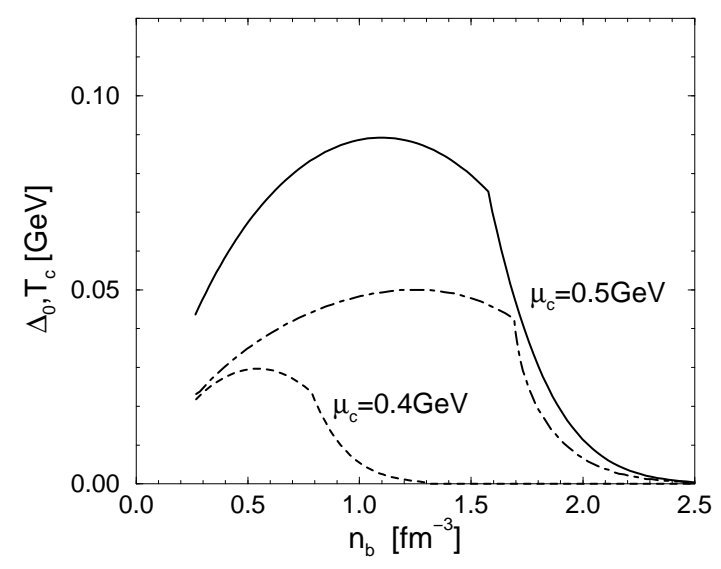

Figure 1. The gap $\Delta(\mu, T=0)$ for $\mu_{c}=0.4,0.5 \mathrm{GeV}$ (dashed and full line, respectively) and critical temperature $T_{c}$ (dashdotted line, $\mu_{c}=0.5 \mathrm{GeV}$ ) versus baryon charge density $n_{b}$.

The situation is different at high density, because new type of condensate, the diquark ones, can be formed. For example, with two flavors, all 4 legs of the instanton vertex can be absorbed by diquark and anti-diquark from a condensate. This means that instanton ensemble may remain to be "random", with the mean field approximation applicable. This is what was done by [3,4] who get a very similar conclusions. The gap and critical temperature are shown in Fig. 1. This condensate is colored, and thus it breaks color $\mathrm{SU}(3)$ to $\mathrm{SU}(2)$ : however it preserves chiral symmetry. In the mean field approximation 
one possible phase, that of chirally asymmetric gas of constituent quarks, is unstable: so quark matter with diquark condensate appears in a first order transition directly from the vacuum. Compared to the original papers mentioned, further work is being made to incorporate the realistic instanton form-factors (see talk by Velkovsky) and include instanton-anti-instanton molecules (see talk by Rapp).

The situation becomes more interesting if one includes the strange quarkf? The instantoninduced interaction becomes a sum of (i) the $4-$ fermion $(\bar{u} u)(\bar{d} d)$ interaction with the coefficient proportional to $m_{s}$ and (ii) the 6 - fermion interaction, with all 3 flavors. If one ignores the former one (or puts $m_{s}=0$ ), there is no way to put all 6 legs into diquark condensates. So we are forced to invent something new.

Alford, Rajagopal and Wilczek (ARW) 50 have decided to abandon instantons and return to the one-gluon exchange (OGE) mechanism[. They have found that the energy minimum corresponds to the phase with "diagonal" locking of color (Latin indices) and flavor (Greek ones)

$$
<q_{i \alpha}^{T} C \gamma_{5} q_{j \beta}>=K_{1} \delta_{i \alpha} \delta_{j \beta}+K_{2} \delta_{i \beta} \delta_{j \alpha}
$$

This phase preserves some diagonal combination of color and flavor, but (in spite of absent $<\bar{q} q>$ ) it is chirally asymmetric. As a result there are massless pions, which can (as usual) be obtained by acting by the chiral generators on the condensate given above. These pions look like diquark rather than quark-anti-quark states, but baryon number is broken anyway.

Let us however return to the instanton-induced 6-fermion interaction. The only ways to get a non-zero answer in the mean field approximation is to put its 6 legs either into the combination of the condensates $<\bar{q} q>^{3}$ (like in vacuum) or into $<q q><\bar{q} \bar{q}><\bar{q} q>$. The latter demands both types of condensates to be present, so we are now forced to break the chiral symmetry. It follows however from the structure of the corresponding gap equations that this phase cannot be present in a weak coupling regime: like the NJL model, this solution exists only above certain critical coupling.

This unwanted feature however disappears when we go outside the usual mean field approximation and include the instanton-anti-instanton "molecules". Let me provide some motivation on why should we do it. In order to explain why these configurations are important, let us compare their contribution with one would typically obtain instead in the mean field (MF) approximation. In both cases we discuss the contribution to the partition function is a second order diagram in the basic 't Hooft interaction, with $\left(2 N_{f}\right)$ fermion lines going between two vertices. In the MF treatment the correlation between color orientations of $I$ and $\bar{I}$ is ignored, so that an averaged coupling is used. Crude estimate of the inaccuracy of this step can be made, even ignoring the orientation dependence of the gauge action (the dipole forces of Callan-Dashen-Gross). Looking at fermionic "hopping" amplitudes one finds that each of them contains explicit factor $\cos (\theta)$,

9 Since the critical $\mu_{c} \sim 300-350 \mathrm{MeV}$ is larger than $m_{s} \approx 140 \mathrm{MeV}$, strange quarks should be included in realistic calculations relevant for neutron stars.

10 The results of ARW are numerically large, suggesting a gap comparable to that induced by instantons. It would be in general surprising to have such strong effect of a perturbative nature, and I hope it will be reduced after further scrutiny. It seems that there was a wrong numerical factor in overall normalization of the coupling constant, which enter in the exponent. 
where $\theta$ is the so called relative orientation angle. The amplitude for "molecules" contains the factor $<(\cos (\theta))^{2 N_{f}}>$, and therefore the integral is dominated by the region close to $\theta=0$, the more so the larger is $N_{f}$. So, the angular integral is much larger than the power of its average value $(1 / 4)^{N_{f}}$ used in the MF.

Clustering of instantons $(I)$ and anti-instantons $(\bar{I})$ into pairs is the simplest possible "ordering". If it happens, quark wave functions get localized (inside these pairs) and chiral symmetry gets restored. Both analytic [20,21] and numerical simulations of the instanton ensemble [22] show that at high $\mathrm{T}$ and/or large $N_{f}$ the ensemble indeed breaks into such objects. In the diquark problem at high density these "molecular" configurations are especially important, because for any value of $N_{f}$ they can provide the desired 4-fermion effective interaction: all "unwanted" $\left(2 N_{f}-4\right)$ lines can in this case be internal ones. If the relative orientation is locked at $\theta=0$ (as explained above), it is described by a simple universal Lagrangian derived and discussed in [17].

The coupling constant (proportional to a density of such molecules) can only be evaluated by some explicit integration and is model dependent. However the structure of the Lagrangian is unique. It is instructive to compare it with the original 't Hooft interaction (for 2 flavors) and OGE. Like two others, it may only contain chirally symmetric operators, and (unlike 't Hooft) it should also respect chiral U(1) symmetry because the "molecules" are topologically trivial. For mesonic channels the effective Lagrangian reads

$$
\begin{array}{r}
L_{\text {molecular }}^{\text {mesonic }}=\frac{2 G}{N_{c}^{2}}\left[\left(\bar{q} \tau^{a} q\right)^{2}-\left(\bar{q} \gamma_{5} \tau^{a} q\right)^{2}\right. \\
\left.\left.+\left(\bar{q} \gamma_{\mu} \gamma_{5} q\right)^{2}-\left(\bar{q} \tau^{a} \gamma_{\mu} \gamma_{5} q\right)^{2} / 4-\left(\bar{q} \tau^{a} \gamma_{\mu} q\right)^{2} / 4\right]+\left(\text { color } r_{o} \text { ctets }\right)\right]
\end{array}
$$

while for color-anti-triplet diquarks it is

$$
\begin{array}{r}
L_{\text {molecular }}^{\underline{3} \text { diquarks }}=\frac{G}{N_{c}\left(N_{c}-1\right)}\left[\left|\left(q^{T} C \gamma_{5} t^{A} \lambda_{A} q\right)\right|^{2}-\left|\left(q^{T} C t^{A} \lambda_{A} q\right)\right|^{2}\right. \\
\left.+\left|\left(q^{T} C \gamma_{\mu} \gamma_{5} t^{A} \lambda_{A} q\right)\right|^{2} / 4-\left|\left(q^{T} C \gamma_{\mu} t^{A} \lambda_{A} q\right)\right|^{2}\right]
\end{array}
$$

Only anti-symmetric flavor and color generators $\lambda_{A}, t_{A}$ are present here (the symmetric part is not shown, it has no scalar diquarks we are interested in.) For $N_{c}=3$ the diquarks are factor 2 weaker, as in the t'Hooft Lagrangian. For comparison, OGE leads to mesonic and diquark Lagrangian to the coefficients $(K / 4)\left(N_{c}^{2}-1\right) / N_{c}^{2}$ in the pion-sigma channels, and $(K / 8)\left(N_{c}+1\right) / N_{c}$ for flavor-color-antisymmetric diquarks and $-(K / 8)\left(N_{c}-1\right) / N_{c}$ for flavor-color-symmetric diquarks. The detailed calculations are not yet finished, but the preliminary result is that a phase with diagonal color-flavor locking (similar to that obtained in [5] using a OGE interaction) happens to be the lowest one.

At very high density instantons are Debye screened by quarks 25. Therefore, one should expect in this limit to return to the OGE interaction and the gaps and the critical temperature in the $\mathrm{MeV}$ range. So if the above-mention conclusion would survive, the whole high density domain would be in the "diagonal" phase.

Whether the simpler (and chirally symmetric) "non-diagonal" phase discussed above for $N_{f}=2$ theory does or does not take place at some intermediate densities depends on the value of the strange quark mass $m_{s}$. If it is big enough, one would have the 2-flavor chirally symmetric phase, with only ud condensate being large. If it is smaller than critical 
value, then we are in the diagonal 3-flavor phase in which chiral symmetry remains broken at any density, without change of phase.

\section{ONE MORE POSSIBLE PHASE, IN WHICH DIQUARKS ARE NOT COOPER PAIRS}

Let us return to the low density limit of real QCD with three colors, ignoring strangeness. (For definiteness, we consider neutron $(u d d)$ matter relevant for stars, in which $u d$ diquarks and $d$ quarks compensate each others color and electric charges.) Let us ask the following question. If one accepts the existence of deeply bound diquarks inside nucleons, they have the smallest energy per unit baryonic charge, and one may ask whether the nuclear matter (made of nucleons) wins energetically over a gas of such diquarks.

The first observation is that Bose condensation of diquarks at zero $\mathrm{T}$ is inevitable, and since scalar diquarks are color anti-triplets, the condensate will select a direction in color space. If we label this direction red $(k=3)$, our $u d$ diquarks are made of blue and green $(k=1,2)$ quarks only. The third quark-type (red $d)$ is basically unaffected, and thus it should form a Fermi gas. So even in the most naive scenario (all diquarks condense in zero momentum state so that their energy is just the sum of masses) one has to pay a price in form of Fermi motion of the 3-rd quark. It is easy to see that it is in fact larger than Fermi motion of nucleons, and so the nuclear matter is indeed the lowest low-density state.

Can a diquark gas still be better at some intermediate density? It is possible to make such naive models in which it is the case. But even with strong binding and including gains due to diquarks Bose character, one cannot definitely answer this question.

Clearly it would be erroneous to conclude that an infinite number of diquarks condenses in the $p=0$ state: diquarks are composite objects and, like nucleons, they should have a short-range repulsion. One may account for repulsion by introducing a scattering length (below $a \simeq 0.3 \mathrm{fm}$ ). Using well known expression for the interacting Bose gas, one can get its energy (per quark)

$$
\frac{\epsilon^{B}}{n_{q}}=\frac{4 \pi a n_{S}}{m}\left(1+\frac{128}{15 \pi^{1 / 2}}\left(a^{3} n_{S}\right)^{1 / 2}\right)
$$

where $n_{S}$ is the scalar diquark density. The first term is the mean field result, and the second term comes from non-condensed diquarks [24]. Results of our calculations are shown in Fig.2, in which "quark-diquark matter" consists of the following components: (i) a Bose gas of $S$ diquarks in chemical equilibrium with (ii) a Fermi gas of blue/green quarks, and neutralized in color and electric charge by an appropriate amount of (iii) red quarks. For definiteness, we use the diquark and quark masses of $500 \mathrm{MeV}$ and $400 \mathrm{MeV}$, respectively.

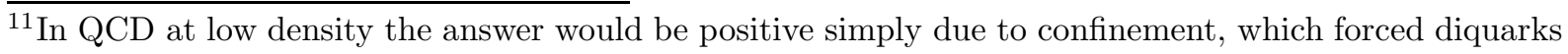
to be connected by color strings. However we study the instanton model, in which the only interaction between quarks is mostly the quasi-local 't Hooft one, and gauge-induced potential is relatively weak.
} 


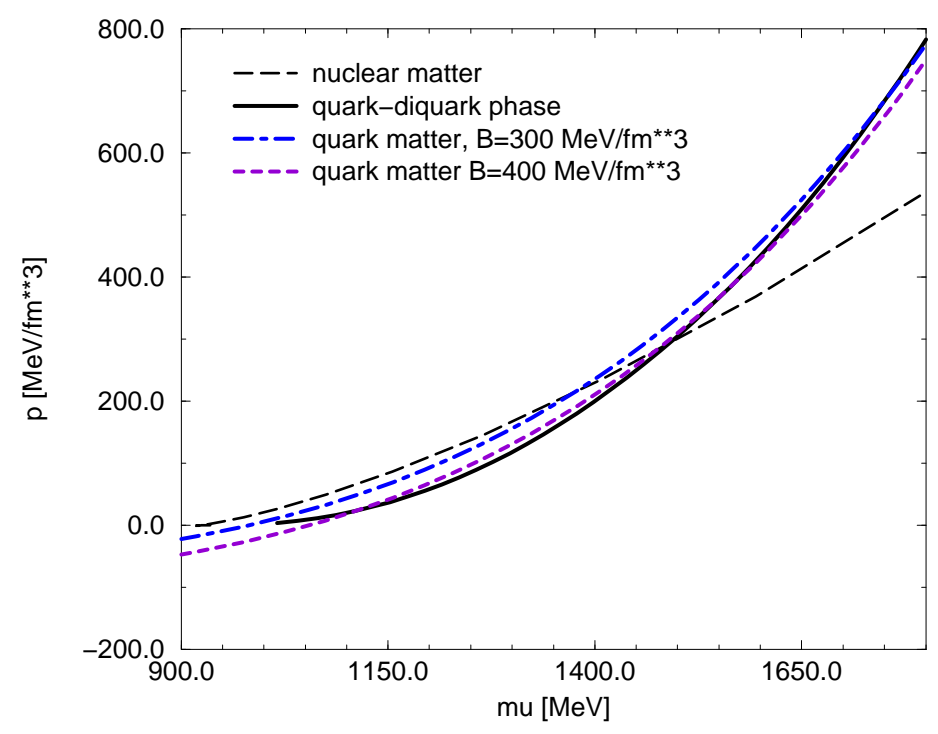

Figure 2. Pressure versus baryonic chemical potential (3 times $\mu$ for quarks used above) for 3 phases: the nuclear matter made of nucleons, the quark-diquark phase made of Bose gas of interacting diquarks and Fermi gas of constituent quarks (no bag constant), and Fermi gas of u,d,s quarks with current masses (with two different bag constants indicated).

\section{SUMMARY}

We have argued that instanton-induced interaction leads to strong color superconductivity in dense matter, with a gap of the order of $100 \mathrm{MeV}$. We think it is unlikely that heavy ion collisions can reach the corresponding part of the phase diagram, and so the main application should be dense stars.

Two different symmetries of the condensates compete: a "non-diagonal" solution, in which some direction in color space is spontaneously selected, or "diagonal" one, in which color is locked with flavor. The former solution preserves chiral symmetry, the second breaks it. It is not yet clear whether we have both of them subsequently, as density grows, or only the second one. The answer depends on numerics, in particularly on the relation between the empirical value of the strange quark mass and its critical value, separating those phases.

One more phase is possible (although this predictions is not robust), containing diquark Bose gas. If it exists, it should separate quark matter from nuclear one. Unfortunately, both this phase and nuclear matter itself can only be discussed with more refined theoretical methods than the mean field analysis applied so far. 


\section{Acknowledgements}

This talk is based on a contuing work done in collaboration with R. Rapp, T. Schäfer and M. Velkovsky. Partial support by the US DOE (grant DE-FG02-88ER40388) is also acknowledged.

\section{REFERENCES}

1. E.V.Shuryak, Phys.Rep. 61, 71, (1980).

2. D. Bailin and A. Love, Phys. Rep. 107, 325 (1984) .

3. R. Rapp, T. Schäfer, E. V. Shuryak and M. Velkovsky, hep-ph/9711396; Phys.Rev.Lett., in press.

4. M. Alford, K. Rajagopal and F. Wilczek, hep-ph/9711395, Phys. Lett. B422 247 (1998).

5. M. Alford, K. Rajagopal and F. Wilczek, hep-ph/9804403.

6. T. Schäfer and E.V. Shuryak, 'Instantons in QCD', hep/ph 9610451, Rev. Mod. Phys. 70, 323 (1998).

7. L. Randall, R. Rattazzi and E.V. Shuryak, hep-ph/9803258.

8. N. Seiberg and E. Witten Nucl.Phys.B426, 19 (1994) .

9. W. Pauli, Nuovo Cimento 6, 205 (1957); F. Gürsey, ibid. 7, 411 (1958).

10. E. Dagotto, F. Karsch and A. Moreo, Phys. Lett. B169 (1986) 421. E. Dagotto, A. Moreo and U. Wolff, Phys. Rev. Lett. 57, 1292 (1986). J.-U. Klaetke and K.-H. Muetter, Nucl. Phys. B342, 764 (1990).

11. T. Schäfer, preprint, hep-ph/9708256.

12. M. C. Chu,J. M. Grandy,S. Huang and J. W. Negele, Phys. Rev. D 48 (1993) 3340.

13. A.B. Migdal et al., Phys. Rep. 192, 179 (1990); D.B. Kaplan and A.E. Nelson, Phys. Lett. B175, 57 (1986); G.E. Brown and H.A. Bethe, Astrophys. J. 423, 659 (1994).

14. E.V. Shuryak, Zh. Eksp. Teor. Fiz. (Sov. Phys. JETP) 74, 408 (1978).

15. M. Hess, F. Karsch, E. Laermann, I. Wetzorke, hep-lat/9804023.

16. R.G. Bettman and L.V. Laperashvili, Yad. Fiz. (Sov. J. of Nucl. Phys.) 41, 463 (1985). E.V. Shuryak and J. Rosner, Phys. Lett. B218, 72 (1989).

17. T. Schäfer, E.V. Shuryak and J.J.M. Verbaarschot, Nucl. Phys. B412, 143 (1994).

18. D. Diakonov, H. Forkel and M. Lutz, Phys. Lett. B373, 147 (1996)

19. L.L. Frankfurt, G.A. Miller and M. Strikman, Ann.Rev.Nucl.Part.Sci.44:501-560,1994, hep$\mathrm{ph} / 9407274$

20. E.-M.Ingelfritz and E.V.Shuryak,Phys.Lett.B325 (1994) 263.

21. M. Velkovsky, E. Shuryak, Phys.Rev.D56, 2766 (1997), hep-ph/9603234.

22. T. Schafer, E.V. Shuryak, Phys.Rev.D53, 6522, (1996), hep-ph/9509337.

23. Fh. de Forcrand, talk at workshop "Instantons and Monopoles", Kopenhagen, June 1998.

24. T.D. Lee and C.N. Yang, Phys. Rev. 105, 1119 (1957).

25. E.V. Shuryak, Nucl. Phys. B203, 140 (1982).

26. M.Stephanov, K.Rajagopal and E.Shuryak,Signatures of the Tricritical Point in QCD, hep$\mathrm{ph} / 9806219$.

27. M. Anselmino et al., Rev. Mod. Phys. 65, 1199 (1993).

28. J. Berges and K. Rajagopal, hep-ph/9804233.

29. M.A. Halasz, A.D. Jackson, R.E. Shrock, M.A. Stephanov and J.J.M. Verbaarschot, hep$\mathrm{ph} / 9804290$. 